\title{
Predicting Retinal Sensitivity Using Optical Coherence Tomography Parameters in Central Serous Chorioretinopathy
}

\section{Satoru Kanda}

The University of Tokyo

Han Peng Zhou

The University of Tokyo

Tatsuya Inoue ( $\nabla$ inouet-tky@umin.ac.jp )

Yokohama City University

Ryosuke Fujino

The University of Tokyo

\section{Aya Sugiura}

The University of Tokyo

\section{Yurika Aoyama}

The University of Tokyo

\section{Kazuaki Kadonosono}

Yokohama City University

Maiko Maruyama-Inoue

Yokohama City University

\section{Ryo Obata}

The University of Tokyo

Ryo Asaoka

The University of Tokyo

\section{Research Article}

Keywords: chronic central serous chorioretinopathy, optical coherence tomography, retinal sensitivity

Posted Date: October 13th, 2021

DOI: https://doi.org/10.21203/rs.3.rs-953689/v1

License: (c) (i) This work is licensed under a Creative Commons Attribution 4.0 International License.

Read Full License 


\section{Abstract \\ Purpose}

To predict the change of retinal sensitivity using optical coherence tomography (OCT) parameters in eyes with central serous chorioretinopathy (CSC).

\section{Methods}

Twenty-three eyes of 23 patients with CSC were enrolled. Retinal sensitivity was measured twice with a microperimetry in all examined eyes. OCT measurement was simultaneously conducted. The relationship between retinal sensitivities and the thicknesses of i) retinal nerve fiver layer + ganglion cell layer (RNFL+GCL), ii) inner nuclear layer (INL) iii) outer nuclear layer (ONL) and iV) serous retinal detachment height (SRDH) were investigated in a point-wise manner. We also investigated the association between the change of retinal sensitivity and OCT parameters at baseline.

\section{Results}

The mean age of participants was $49.8 \pm 10.7$ years. The mean SRDH was significantly lower $(p<0.001)$ and the mean retinal sensitivity $(p<0.001)$ was significantly higher in the 2 nd examination compared to the 1st measurements, however LogMAR visual acuity (VA) was not significantly different between the two measurements $(p=0.063)$. LogMAR VA was associated with retinal sensitivity both in the 1 st and 2nd measurements $(p<0.001)$. The retinal sensitivity in the 2 nd examination was significantly correlated with retinal sensitivity, RNFL+GCL, INL, ONL, SRDH in the 1st examination and the improvement of SRDH.

\section{Conclusions}

Retinal sensitivity was associated with retinal structure in eyes with CSC; these parameters were useful to predict the change of visual function before the treatment.

\section{Introduction}

Central serous chorioretinopathy (CSC) is characterized by serous retinal detachment (SRD) in the macula accompanied with dysfunction of the retinal pigment epithelium (RPE). [1-3] Although CSC generally resolves spontaneously, some patients complain metamorphopsia and central scotoma even after the resolution of SRD; thus, it is important to evaluate not only the visual acuity, but also the retinal sensitivity, or visual field (VF) [4-6]. In particular, the microperimetry, also known as fundus related perimetry, is a useful tool in assessing central retinal sensitivity as it is able to evaluate the measurement of retinal sensitivity at an exact location through the fundus image tracking. [7-16] 
Indeed, we previously reported that SRD height (SRDH) was closely correlated with the mean retinal sensitivity measured with the MP-3 microperimetery (NIDEK, Co.Ltd, Japan) in CSC eyes with SRD. [12] However, this study was cross-sectional and RS was analyzed merely as the mean of whole field, rather than in the point-wise manner. Therefore, the present study enrolled CSC patients who demonstrated the improvement of SRD during the follow-up period and we measured retinal sensitivity twice in eyes with CSC, and investigated whether visual acuity and the mean retinal sensitivity were associated with the OCT parameters at each measurement time. Furthermore, we analyzed whether the visual function can be predicted using the OCT parameters at baseline.

\section{Methods}

This study was a retrospective observational case series and was approved by the Research Ethics Committee of the Graduate School of Medicine and Faculty of Medicine at The University of Tokyo. Written informed consent was given by all participants and our study was conducted in accordance with the tenets of the Declaration of Helsinki.

\section{Subject}

Twenty-three eyes of 23 patients (18 male, 5 female) with chronic CSC were enrolled in the current study. The mean age of participants was $49.8 \pm 10.7$ years (mean \pm standard deviation: SD). All patients underwent comprehensive ophthalmic examinations, including refractive error, best-corrected visual acuity (BCVA), intraocular pressure, anterior segment examination, and fundus biomicroscopy with pupil dilation. Patients were diagnosed based on OCT, fluorescein angiography (FA) and Indocyanine green angiography (ICGA). Chronic CSC was defined as those with CSC lasting more than 3 months. At the 1 st measurement, all enrolled eyes demonstrated subretinal fluid (SRD) within central 12 degrees which decreased at the 2nd examination. The exclusion criteria were as follow: (1) a history of other retinal disorders; (2) a history of laser photocoagulation or photodynamic therapy; (3) the presence of high myopia (-6.0 diopter or greater)

\section{VF measurement}

VF measurement was carried out using the MP-3 microperimetry. The MP-3 microperimeter test is based on the 4-2 full threshold strategy using the Goldmann III stimulus size, as previously described. [13,17] The 25 measurement points were located within 12 degrees of the macular area as shown in Fig.1. All patients underwent VF measurement both at the 1st and 2nd measurements.

\section{OCT measurement}

Spectral domain (SD) OCT images were obtained using the Spectralis OCT (Heidelberg Engineering, Germany). All OCT images consisted of line scans (horizontal and vertical B-scans), raster scans, and topographic mapping. The raster scan was performed using $25 \mathrm{~B}$-scans (768 A-scans per B-scan) in a $30 \times 20$-degree area. OCT measurements were also performed both at the 1 st and 2 nd measurements in 
each eye. Using OCT images, we measured total retinal thickness (RT), retinal nerve fiber layer + ganglion cell layer (RNFL+GCL), inner nuclear layer (INL), outer nuclear layer (ONL), and SRDH at the 25 locations corresponding to 25 measurement points with MP-3. RT was defined as the distance between the internal limiting membrane and the RPE. SRDH was defined as the distance between ellipsoid zone (EZ) and RPE. These data were collected independently by two examiners. If the difference was large between two examiners, a panel discussion was held to draw a conclusion.

\section{Statistical analyses}

LogaMAR VA and mean retinal sensitivity were compared between the 1st and 2nd examinations (VA1 and VA2, $m R S 1$ and $m R S 2$ ). Retinal sensitivities, thicknesses (RT, RNFL+GCL, INL, and ONL), and also SRDH were compared between the 1st and 2nd examinations (RS1 and RS2, RT1 and RT2, RNFL+GCL1 and RNFL+GCL2, INL1 and INL2, ONL1 and ONL2, SRDH1 and SRDH2), at 25 measurement points. Among 6 variables (age, RNFL+GCL1, INL1, ONL1, SRDH1 and the improvement in SRDH: dSRDH), the variables associated with the retinal sensitivity at the 2 nd examination were identified, using the corrected Akaike Imformation Criterion (AICC) model selection; the optimal model was selected from $2^{6}$ patterns. [18] The AICc is a well-known statistical measure with which optimal variables can be determined without having an over-fit problem, unlike the coefficient of determination. [19] All statistical analyses were performed using the statistical programming language ' $R$ ' ( $R$ version 3.1.3; The Foundation for Statistical Computing, Vienna, Austria).

\section{Results}

Baseline characteristics of the current study are summarized in Table 1. The mean SRDH at the1st and 2nd examinations (mSRDH1 and $\mathrm{mSRDH} 2$ ) were 31.0 $\pm 30.7 \mu \mathrm{m}$ and $6.6 \pm 14.1 \mu \mathrm{m}$, respectively, and there was a significant difference between $\mathrm{mSRDH} 1$ and $\mathrm{mSRDH} 2(\mathrm{p}<0.001$, Wilcoxon signed rank test). Among 23 eyes, 13 eyes demonstrated a complete resolution of SRD within central 12 degrees at the 2nd examination. The improvement of SRD was observed in residual 10 eyes during observation period. There was no significant difference between VA1 and VA2, although the $p$ value approached significance $(p=0.063$, Wilcoxon signed rank test). On the other hand, a significant improvement was observed in mRS2 compared to mRS1 ( $<<0.001$, Wilcoxon signed rank test). mRS1 was significantly correlated with $V A 1$, and $m R S 2$ was also significantly related to VA2 ( $<<0.001, p=0.0030$, linear regression analysis). dSRDH (SRDH2 - SRDH1) was $-24.5 \pm 44.3 \mu \mathrm{m}$.

There were no significant relationships between VA1 and $\mathrm{mSRDH} 1$ ( $p=0.76$, linear regression analysis), and between VA2 and mSRDH2 ( $p=0.43)$. On the other hand, mRS1 was significantly associated with mSRDH1 $(p=0.041)$ and mRS2 was also associated with mSRDH2 $(p<0.001)$.

In the pointwise analysis ( $25 \times 23$ measurement points), RT1 and RT2 were $343.1 \pm 61.3$ and $309.1 \pm 46.4$ $\mu \mathrm{m}, \mathrm{RNFL}+\mathrm{GCL} 1$ and RNFL+GCL2 were $67.2 \pm 22.2$ and $66.3 \pm 22.6 \mu \mathrm{m}$, INL1 and INL2 were $39.0 \pm 11.7$ and $37.6 \pm 11.5 \mu \mathrm{m}, \mathrm{ONL} 1$ and ONL2 were $44.6 \pm 20.5$ and $49.7 \pm 21.1 \mu \mathrm{m}$, and SRDH1 and SRDH2 were 
$31.0 \pm 52.1$ and $6.6 \pm 21.4 \mu \mathrm{m}$, respectively. Significant differences were observed between RT1 and RT2, INL1 and INL2, ONL1 and ONL2, SRDH1 and SRDH2 ( $<<0.001$, respectively, Wilcoxon signed rank test) but not between RNFL+GCL1 and RNFL+GCL2 $(p=0.20)$.

As a result of model selection, the optimal model for RS2 was as follows:

$\mathrm{RS} 2=22.9-0.053 \times$ age (standard error: $\mathrm{SE}=0.026)+0.21 \times \mathrm{RS} 1(\mathrm{SE}=0.027)-0.0099 \times \mathrm{RNFL}+\mathrm{GCL} 1$ $(\mathrm{SE}=0.0064)+0.027 \times \mathrm{INL} 1(\mathrm{SE}=0.012)+0.021 \times \mathrm{ONL} 1(\mathrm{SE}=0.0070)-0.070 \times \mathrm{SRDH} 1(\mathrm{SE}=0.0066)-$ $0.067 \times$ dSRDH $(S E=0.0072)(\mathrm{AICc}=2749.4)$

\section{Discussion}

In the current study, MP-3 and OCT measurements were performed in patients with chronic CSC. The results show that the mean SRD height was significantly associated with retinal sensitivity but not with VA. We also found that the retinal sensitivity at the 2 nd examination was significantly related to retinal structural parameters (the thicknesses of RNFL+GCL, ONL, and INL and also SRDH) at the 1st examination, suggesting that it was useful to use the baseline OCT parameters when predicting the retinal sensitivity after the resolution of SRD in eyes with CSC.

In the clinical settings, VA measurement is most frequently used when evaluating visual function in macular diseases, including CSC. However, we recently reported the benefit of using retinal sensitivity (visual field) in CSC eyes with SRD as retinal sensitivity was significantly associated with SRDH, whereas logMAR VA was not. [12] Similarly, our current study suggested no significant relationships were observed between VA1 and mSRDH1 and between VA2 and mSRDH2; on the other hand, there were significant associations between mRS1 and mSRDH1 and between mRS2 and mSRDH2.

In the point-wise analysis, RNFL+GCL1, ONL1, INL1, SRDH1 and dSRDH in addition to RS1 were significantly associated with RS2. In other words, these variables were useful to predict retinal sensitivity after the resolution of SRD in eyes with CSC. Among the OCT parameters, ONL1 and INL1 were positively correlated with RS2, on the other hand RNFL+GCL1 was negatively associated. This result is somewhat contradicting because the RNFL+GCL thickness is thought to be positively correlated with the retinal sensitivity in glaucoma. Considering that the coefficient value of RNFL+GCL1 was much less compared with those of ONL1 and INL1, it was possible that RNFL+GCL1 was a negligible variable for RS2.

Several studies indicated that the integrity of EZ was correlated with visual function in macular diseases. We also reported that residual EZ index was correlated with visual acuity and the retinal sensitivity in resolved CSC. [20] In our present study, EZ disruption was not considered as it can be difficult to identify the $E Z$ line in detached retina. Instead, we measured the ONL thickness in conjunction with RNFL+GCL, INL and SRDH in each measurement point and as a result, these were correlated with retinal sensitivity.

There were some limitations in the current study. First, this study was retrospective and the patients who had undergone the treatment such as laser photocoagulation were excluded. It would be of interest to 
investigate the accuracy of prognosis prediction after the treatment in such eyes in the future. Second, in the present study, fundus autofluorescence (AF) measurement was not taken into consideration. The short-wavelength AF signal was reported to be associated with retinal sensitivity in eyes with chronic CSC. [21] Besides, we also recently reported that AF measurement, especially near-infrared AF, was superior to OCT measurement (EZ disruption) for detecting the deterioration of retinal sensitivity in eyes with chronic CSC. [22] A further study would be needed to investigate whether the AF measurement enables a more accurate prediction of visual function.

In conclusion, retinal sensitivity is more reflective of the retinal structure in eyes with chronic CSC. Measuring the retinal sensitivity at baseline is important for predicting visual outcome in eyes with CSC.

\section{Declarations}

\section{Ethics approval and consent to participate:}

This study was approved by the Research Ethics Committee of the Graduate School of Medicine and Faculty of Medicine at The University of Tokyo. The committee's reference number is 2217.

Consent for publication: Done

Availability of data and material: None

Competing interests: Ryo Asaoka is an Editorial board member for BMC Ophthalmology.

Funding: None

Authors' contributions: SK and TI were responsible for writing the protocol and report, conducting the search, screening potentially eligible studies, extracting and analyzing data, interpreting results, updating reference lists and creating 'Summary of findings' tables. HZ, RF, AS, YA, KK, MM, RO and RA provided feedback on the report. All authors have read and approved the manuscript.

Running head: Retinal sensitivity and retinal structure in chronic CSC

\section{References}

1. Daruich A, Matet A, Dirani A, Bousquet E, Zhao M, Farman N, et al. Central serous chorioretinopathy: RECENT findings and new physiopathology hypothesis. Prog Retin Eye Res. 2015; 48: 82-118.

2. Ojima $Y$, Hangai $M$, Sasahara $M$, Gotoh $N$, Inoue $R$, Yasuno $Y$, et al. Tree-dimensional imaging of the foveal photoreceptor layer in central serous chorioretinopathy using high-speed optical coherence tomography. Ophthalmology. 2007; 114: 2197-2207.

3. Vogel RN, Langlo CS, Scoles D, Carroll J, Weinberg DV, Kim JE. High-resolution imaging of intraretinal structures in active and resolved central serous chorioretinopathy. Invest Ophthalmol Vis Sci. 2017; 58: 42-49. 
4. Wang M, Sander B, Cour ML, Larsen M. Clinical characteristics of subretinal deposits in central serous chorioretinopathy. Acta Ophthalmol Scand 2005; 83: 691-696.

5. Gackle HC, Lang GE, Freissler KA, Lang GK. Central serous chorioretinopathy. Clinical, fluorescein angiography and demographic aspects. Ophthalmologe 1998; 95: 529-533.

6. Baran NV, Gurlu VP, Esgin H. Long-term macular function in eyes with central serous chorioretinopathy. Clin Exp Ophthalmol 2005; 33: 369-372.

7. Ozdemir H, Karacorlu SA, Senturk F, Karacorlu M, Uysal O. Assessment of macular function by microperimetry in unilateral resolved central serous chorioretinopathy. Eye (Lond) 2008; 22: 204208.

8. Ehrlich R, Mawer NP, Mody CH, Brand CS, Squirrell D. Visual function following photodynamic therapy for central serous chorioretinopathy: a comparison of automated macular microperimetry versus best-corrected visual acuity. Clin Exp Ophthalmol 2012; 40: e32-39.

9. Reibaldi M, Boscia F, Avitabile T, Uva MG, Russo A, Zagari M, et al. Functional retinal changes measured by microperimetry in standard-fluence vs low-fluence photodynamic therapy in chronic central serous chorioretinopathy. Am J Ophthalmol. 2011;151; 953-960. e2.

10. Ojima $Y$, Tsujikawa A, Hangai $M$, Inoue $R$, Sakamoto A, Yoshimura N, et al. Retinal sensitivity measured with the micro perimeter 1 after resolution of central serous chorioretinopathy. Am $\mathrm{J}$ Ophthalmol. 2008; 146: 77-84.

11. Springer $C$, Volcker HE, Rohrschneider K. Central serous chorioretinopathy-retinal function and morphology: microperimetry and optical coherence tomography. Ophthalmologe 2006; 103: 791797.

12. Sugiura A, Fujino R, Takemiya N, Simizu K, Matsuura M, Murata $H$, et al. The association between visual function and retinal structure in chronic central serous chorioretinopathy. Sci Rep. 2017; 7: 16288.

13. Matsuura M, Murata H, Fujino $Y$, Hirasawa $K$, Yanagisawa $M$, Asaoka R. Evaluating the usefulness of MP-3 microperimetry in glaucoma patients. Am J Ophthalmol. 2018; 187: 1-9.

14. Asahina $Y$, Kitano $M$, Hashimoto $Y$, Yanagisawa $M$, Murata $H$, Inoue $T$, et al. The structure-function relationship measured with optical coherence tomography and a microperimeter with autotracking: the MP-3, in patients with retinitis pigmentosa. Sci Rep. 2017; 7: 15766.

15. Fujino R, Asaoka R, Aoki S, Sugiura A, Kusakabe M, Asano-Shimizu K, et al. The usefulness of the retinal sensitivity measurement with a microperimetry for predicting the visual prognosis of branch retinal vein occlusion with macular edema. Graefes Arch Clin Exp Ophthalmol. 2020; 258(9): 19491958.

16. Lee C, Baughman DM, Lee AY. Deep learning is effective for the classification of OCT images of normal versus Age-related Macular Degeneration. Ophthalmol Retina. 2017; 1(4): 322-327.

17. Igarashi N, Matsuura M, Hashimoto $Y$, Hirasawa $K$, Murata $H$, Inoue $T$, et al. Assessing visual fields in patients with retinitis pigmentosa using a novel microperimeter with eye tracking: the MP-3. PLoS One. 2016; 11: e0166666. 
18. Chung HW, Yun CM, Kim JT, Kim SW, Oh J, Huh K. Retinal sensitivity assessed by microperimetry and corresponding retinal structure and thickness in resolved central serous chorioretinopathy. Eye (Lond). 2014; 28: 1223-1230.

19. Nakagawa S, Schielzeth $\mathrm{H}$. A general and simple method for obtaining R2 from generalized linear mixed-effects models. Methods Ecol Evol. 2013; 4(2): 133-142.

20. Fujita A, Aoyama Y, Tsuneyoshi S, Sugiura A, Azuma K, Asano-Shimizu K, et al. Association between visual function and the integrity of residual ellipsoid zone in resolved central serous chorioretinopathy. Sci Rep. 2019; 9: 12433.

21. Kim SK, Kim SW, Oh J, Huh K. Near-infrared and short wavelength autofluorescence in resolved serous chorioretinopathy: association with outer retinal layer abnormalities. Am J Ophthalmol. 2013; 156(1): 157-164.

22. Soga $H$, Asaoka R, Kadonosono $K$, Maruyama-Inoue $M$, Igarashi N, Kitano M, et al. Association of Near-Infrared and Short-Wavelength Autofluorescence With the Retinal Sensitivity in Eyes With Resolved Central Serous Chorioretinopathy. Invest Ophthalmol Vis Sci. 2021; 62(3): 36.

\section{Tables}

Table 1

Subjects demographics.

\begin{tabular}{|ll|}
\hline Variables & Mean \pm SD [range] \\
\hline Age (years) & $49.8 \pm 10.5$ [26 to 78] \\
\hline Gender, male: female & $18: 5$ \\
\hline Eye, right: left & $10: 13$ \\
\hline LogMAR VA at baseline & $0.038 \pm 0.17$ [-0.18 to 0.52] \\
\hline LogMAR VA at 2nd examination & $0.012 \pm 0.17$ [-0.18 to 0.52] \\
\hline mRS at baseline (dB) & $23.7 \pm 5.73$ [0 to 34] \\
\hline mRS at 2nd examination (dB) & $26.2 \pm 3.76$ [0 to 32] \\
\hline $\begin{array}{l}\text { SD: standard deviation, Log MAR VA: logarithm of the minimal angle of resolution visual acuity, mRS: } \\
\text { mean retinal sensitivity, RNFL: retinal nerve fiber layer, GCL: ganglion cell layer, INL: inner nuclear layer, } \\
\text { ONL: outer nuclear layer, SRDH: serous retinal detachment height }\end{array}$ \\
\hline
\end{tabular}


Table 2

OCT measurements at 1 st and 2 nd examinations on all measurement points.

\begin{tabular}{|c|c|c|c|}
\hline \multirow[t]{2}{*}{ Variables } & \multicolumn{2}{|l|}{ Mean \pm SD [range] } & \multirow[t]{2}{*}{$\mathrm{p}$ value } \\
\hline & 1st examination & 2nd examination & \\
\hline RT & $343.1 \pm 61.2[151$ to 531$]$ & $309.1 \pm 46.4[32$ to 445$]$ & $<0.001$ \\
\hline RNFL+GCL & $67.2 \pm 22.2[0$ to 132$]$ & $66.3 \pm 22.6[0$ to 120$]$ & 0.20 \\
\hline INL & $39.0 \pm 11.6$ [0 to 78$]$ & $37.6 \pm 11.5$ [0 to 68$]$ & $<0.001$ \\
\hline ONL & $44.6 \pm 20.5[3$ to 125$]$ & $49.7 \pm 21.1[4$ to 120$]$ & $<0.001$ \\
\hline SRDH & $31.0 \pm 52.1[0$ to 230$]$ & $6.6 \pm 21.4[0$ to 144$]$ & $<0.001$ \\
\hline \multicolumn{4}{|c|}{$\begin{array}{l}\text { There were significant differences between two examinations in RT, INL, ONL and SRDH }(p<0.001 \text {, } \\
\text { respectively, Wilcoxon signed rank test). On the other hand, no significant difference was observed in } \\
\text { RNFL+GCL }(p=0.20) \text {. }\end{array}$} \\
\hline \multicolumn{4}{|c|}{$\begin{array}{l}\text { SD: standard deviation, RT: retinal thickness, RNFL: retinal nerve fiber layer, GCL: ganglion cell layer, } \\
\text { INL: inner nuclear layer, ONL: outer nuclear layer, SRDH: serous retinal detachment height }\end{array}$} \\
\hline
\end{tabular}

Table 3

The AICc model selection for RS at the 2nd examination (RS2).

\begin{tabular}{|llll|}
\hline & Estimate & Standard error & p value \\
\hline age & -0.053 & 0.026 & $<0.001$ \\
\hline RS1 & 0.21 & 0.027 & $<0.001$ \\
\hline RNFL+GCL1 & -0.0099 & 0.0064 & 0.002 \\
\hline INL1 & 0.027 & 0.012 & 0.39 \\
\hline ONL1 & 0.021 & 0.0070 & 0.051 \\
\hline SRDH1 & -0.070 & 0.0066 & $<0.001$ \\
\hline dSRDH & -0.067 & 0.0072 & $<0.001$ \\
\hline $\begin{array}{l}\text { The optimal model for RS2 included age, RS1, RNFL+GCL1, INL1, ONL1, SRDH1 and dSRDH } \\
\text { (AICc=2749.4). }\end{array}$ & & \\
\hline $\begin{array}{l}\text { AlCc: corrected Akaike Imformation Criterion, RS: retinal sensitivity, RNFL: retinal nerve fiber layer, } \\
\text { GCL: ganglion cell layer, INL: inner nuclear layer, ONL: outer nuclear layer, SRDH: serous retinal } \\
\text { detachment height, dSRDH: the change of SRDH (SRDH2-SRDH1) }\end{array}$ & \\
\hline
\end{tabular}

\section{Figures}




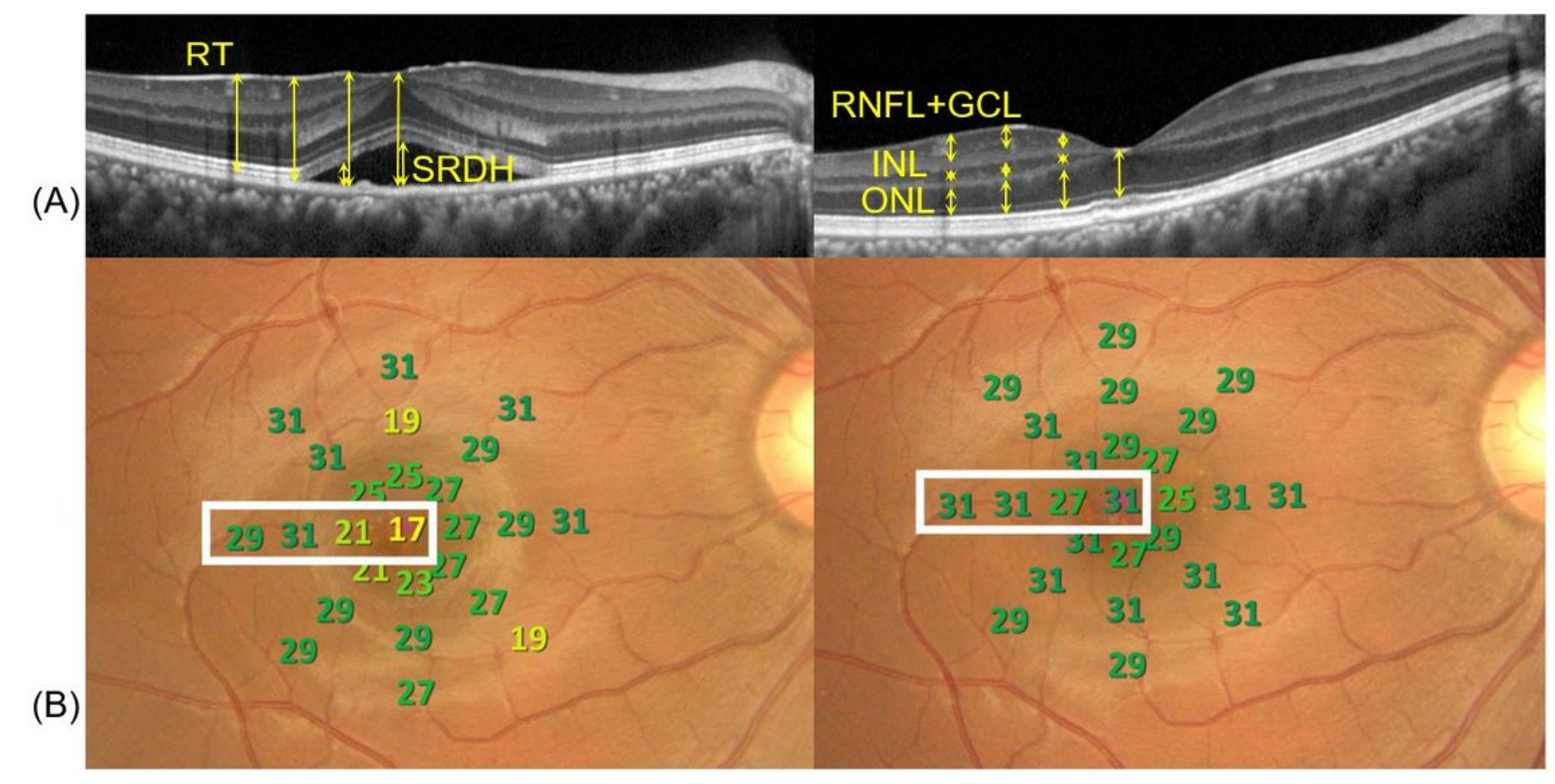

\section{Figure 1}

Representative case in an eye with chronic CSC. Retinal sensitivity was measured at the 1st and 2nd examination (after the SRD spontaneously resolved). (A) OCT parameters were measured at each point. Yellow arrows indicate retinal structures at each measurement point. (B) Retinal sensitivity measured with the MP-3 microperimeter. Retinal sensitivity was measured within 12 degrees in CSC. Notably, the 4 measurement points in the upper and lower images correspond each other. RT: retinal thickness, RNFL+GCL: retinal nerve fiber layer + ganglion cell layer, INL: inner nuclear layer, ONL: outer nuclear layer, SRDH: serous retinal detachment height, OCT: optical coherence tomography, CSC: central serous chorioretinopathy 